\title{
Nível de estresse e humor em estudantes de Educação Físicano último ano de graduação
}

\author{
Nivel de estrés y de humor en estudiantes en estudiantes \\ de Educación Física en el último año de graduación
}

\author{
Stress and mood level in Physical Education students \\ in the last year of graduation
}

\section{${ }^{1}$ Fernanda Alves de Souza, ${ }^{2}$ João Gabriel Miranda de Oliveira, ${ }^{3}$ Juliana Brandão Pinto de Castro, ${ }^{4}$ Dirceu Ribeiro Nogueira da Gama, \& ${ }^{5}$ Vicente Pinheiro Lima}

Souza, F., Oliveira, J., Castro, J., Gama, D., \& Lima, V. (2022). Nível de estresse e humor em estudantes de Educação Física no último ano de curso. Revista Ciencias de la Actividad Física UCM, 23(1), enero-junio, 1-12. https://doi.org/10.29035/rcaf.23.1.2

RESUMO

Introdução: As dificuldades e a pressão do último ano da graduação podem afetar negativamente a saúde mental de estudantes universitários.

Objetivo: Verificar o nível de estresse e humor em estudantes de Educação Física no último ano de graduação.

Métodos: A amostra foi composta por 38 estudantes (idade: 25,51 \pm 4,24 anos), concluintes do curso de Educação Física. Para análise do nível de estresse e humor foram utilizados os questionários da Escala de Percepção de Estresse PSS14 e a Escala de humor de Brunel, respectivamente. Resultados: O teste inferencial não paramétrico de Friedman verificou que há diferença significativa ( $p<0,001)$ entre os níveis de estresse: Nunca (4,94\%), Quase nunca (15,02), Às vezes (23,87), Pouco frequente $(23,46)$ e Muito frequente $(32,72)$ e entre as respostas ao questionário de humor ( $p=0,026)$ com: Nada (38,27), Pouco $(17,65)$, Moderadamente $(16,23)$, Bastante $(14,36)$ e extremamente $(13,49)$. Conclusão: Diante dos resultados da investigação, concluímos que os participantes do estudo se apresentaram em condições de estresse, entretanto não expuseram perda de humor.

Palavras chave: Autoavaliação, Estresse Psicológico, Transtornos do humor, Inquéritos e Questionários, Saúde do Estudante.

Grupo de Pesquisa em Biodinâmica do Desempenho, Exercício e Saúde (BIODESA), Universidade Castelo Branco,Rio de Janeiro, Brasil. https://orcid.org/0000-0002-2424-6860 | nanda10rj@hotmail.com

2 Grupo de Pesquisa em Biodinâmica do Desempenho, Exercício e Saúde (BIODESA), Universidade Castelo Branco,Rio de Janeiro, Brasil. https://orcid.org/0000-0002-2957-7079| professorjoaogabrielmdo@gmail.com

3 Grupo de Pesquisa em Biodinâmica do Desempenho, Exercício e Saúde (BIODESA), Universidade Castelo Branco. Laboratório do Exercício e do Esporte (LABEES). Programa de Pós-graduação em Ciências do Exercício e do Esporte, Universidade do Estado do Rio de Janeiro, Rio de Janeiro, Brasil. https://orcid.org/0000-0002-5656-0782 | julianabrandaoflp@hotmail.com

4 Laboratório do Exercício e do Esporte (LABEES). Programa de Pós-graduação em Ciências do Exercício e do Esporte, Universidade do Estado do Rio de Janeiro, Rio de Janeiro, Brasil. https://orcid.org/0000-0003-4972-650X | dirceurng@gmail.com

5 Grupo de Pesquisa em Biodinâmica do Desempenho, Exercício e Saúde (BIODESA), Universidade Castelo Branco. Laboratório do Exercício e do Esporte (LABEES). Programa de Pós-graduação em Ciências do Exercício e do Esporte, Universidade do Estado do Rio de Janeiro, Rio de Janeiro, Brasil. https://orcid.org/0000-0002-7534-265X | professorvicentelima@gmail.com 
Souza, F., Oliveira, J., Castro, J., Gama, D., \& Lima, V. (2022). Nível de estresse e humor em estudantes de Educação Física no último ano de curso. Revista Ciencias de la Actividad Física UCM, 23(1), enero-junio, 1-12. https://doi.org/10.29035/rcaf.23.1.2

\section{RESUMEN}

Introducción: Las dificultades y la presión del último año de graduación pueden afectar negativamente la salud mental de los estudiantes universitarios.

Objetivo: Verificar el nivel de estrés y estado de humor en los estudiantes de Educación Física en su último año de graduación. Métodos: La muestra consistió en 38 estudiantes (25,51 \pm 4,24 años), graduados del curso de Educación Física. Para el análisis del nivel de estrés y estado de ánimo, se utilizaron los cuestionarios de la Escala de percepción del estrés PSS14 y la escala del estado de ánimo de Brunel, respectivamente.

Resultados: La prueba inferencial no paramétrica de Friedman encontró que existe una diferencia significativa ( $p<0,001)$ entre los niveles de estrés Nunca (4,94\%), Casi nunca $(15,02)$, A veces $(23,87)$, Poco común $(23,46)$ y Muy frecuente $(32,72)$ y entre las respuestas al cuestionario de estado de ánimo ( $p=0,026)$ con Ninguno $(38,27)$, Poco (17,65), Moderado (16,23), Bastante $(14,36)$ y extremadamente $(13,49)$. Conclusión: en vista de los resultados de la investigación, concluimos que los participantes del estudio estaban en condiciones de estrés, sin embargo, no mostraron pérdida de humor.

Palabras clave: Autoevaluación, estrés psicológico, trastornos del humor, encuestas y cuestionarios, salud del estudiante.

\section{ABSTRACT}

Introduction: The difficulties and pressure of the last year of graduation can negatively affect the mental health of university students.

Objective: To verify the level of stress and mood in Physical Education students in the last year of graduation.

Methods: The sample consisted of 38 students (age: 25,51 \pm 4,24 years), graduating from the Physical Education course. Stress Perception Scale questionnaires PSS14 and Brunel's mood scale, respectively, were used to analyze the level of stress and mood. Results: Friedman's non-parametric inferential test found that there is a significant difference $(p<0,001)$ between stress levels: Never (4,94\%), Almost Never $(15,02)$, Sometimes $(23,87)$, Uncommon $(23,46)$ and Very frequent $(32,72)$ and among the responses to the mood questionnaire $(p=0,026)$ with: Nothing (38,27), Little (17,65), Moderately (16,23), Quite $(14,36)$ and Extremely $(13,49)$. Conclusion: In view of the results of the investigation, we concluded that the study participants were in conditions of stress, however they did not exhibit loss of mood.

Key words: Self-Assessment, Stress, Psychological, Mood Disorders, Surveys and Questionnaires, Student Health.

\section{INTRODUÇÃO}

Estudos têm evidenciado altos níveis de estresse em estudantes universitários decorrentes de diversos fatores, como sobrecarga acadêmica, rede de amizades, expectativas sociais e dificuldades financeiras (Castro et al., 2017, Saleh et al., 2017, Ng et al., 2016). Esse alto nível de estresse desencadeia fatores fisiológicos no indivíduo que, de forma prolongada, podem ocasionar problemas à saúde (Yaribeygi et al., 2017). Ademais, no âmbito psicológico, o estresse tem sido relacionado a fatores como ansiedade, ataques de pânico e depressão (Shankar \& Park, 2016).
Alunos que exibem maior estresse autopercebido parecem apresentar pior performance acadêmica (Frazier et al., 2019). Asayesh et al. (2016) conduziram um estudo com alunos de enfermagem reforçando essa constatação, cujos resultados mostraram que quanto maior o nível de estresse, pior o desempenho acadêmico. Resultados semelhantes foram encontrados por Gustems-Carnicer et al. (2019), nos quais os estudantes de cursos de formação de professores que apresentaram maiores 
níveis de estresse também apresentaram baixo desempenho acadêmico.

O declínio no desempenho acadêmico pode advir da relação estresse aprendizado (Kluen et al., 2017, Lee, 2017). Isso decorre da ativação induzida por estresse, que faz com queo eixo hipotálamohipófise-adrenal (HPA) ative estruturas, como o núcleo paraventricular (PVN), a glândula pituitária e o tecido adrenal. A partir dessa ativação, tem-se a produção de hormônios relacionados ao estresse, como o hormônio liberador de corticotropina ( $\mathrm{CRH})$, hormônio adrenocorticotrópico (ACTH) e corticosterona (CRT), os quais parecem interferir em estruturas relacionadas ao aprendizado (Lindau et al., 2016)

Outro fator também relacionado ao desempenho acadêmico é o humor. De acordo com os achados de Huang \& Lee (2019), o humor está relacionado à inteligência emocional e a maior satisfação com a vida. Quando utilizado de maneira eficaz, o humor parece ser benéfico para relacionamentos e para aprimorar o desempenho. Especificamente, os benefíciosdo humor são relacionados ao desempenho acadêmico por atrair e sustentar a atenção do aluno, reduzir a ansiedade e aumentar a motivação e a participação na aula. Além disso, também estimula diversos sistemas fisiológicos que diminuem os níveis de hormônios relacionados ao estresse, como o cortisol e a epinefrina, e aumentam a ativação do sistema de recompensa dopaminérgico mesolímbico (Savage et al., 2017).

Segundo a teoria neurobiológica do afeto positivo, o humor pode beneficiar o desempenho cognitivo devido ao aumento de dopamina no cérebro. Porém, devem-se considerar as diferenças individuais que podem influenciar na hipótese dessa teoria, dado que indivíduos com boa capacidade de memória de trabalho aparentam não se beneficiar da indução positiva do humor tal como os indivíduos cuja capacidade de memória de trabalho é baixa (Greeley \& Seidler, 2017). Adicionalmente, o humor parece servir como uma representação de um momento que varia entre diferentes estados ao longo do tempo e, com essa variação, a representação pode refletir em otimismo ou pessimismo (Eldar et al., 2016). Sendo assim, não há certeza de que o humor pode interferir positivamente no aprendizado. Contanto, sabe-se que indivíduos induzidos a estímulos de humor positivo podem apresentar melhorestaxas de aprendizado do que indivíduos não submetidos a esses estímulos (Lujan \& DiCarlo, 2016).

Partindo do pressuposto de que o estresse pode estar presente durante todo o percurso acadêmico, havendo apenas uma variação nos níveis de acordo com o período de curso (Moutinho et al., 2017) e de que o humor positivo pode proporcionar ao indivíduo um ambiente mais propício para um bom desempenho acadêmico (Tsukawaki \& Imura, 2020), o presente estudo buscou verificar o nível de estresse e de humor em estudantes de Educação Física no último ano de graduação.

\section{MÉTODOS}

\section{Desenho de estudo e amostra}

○ presente estudo caracteriza-se como uma pesquisa descritiva com uma abordagem quantitativa (Thomas et al., 2012). A amostra foi composta por estudantes do último ano do curso de graduaçãoem bacharelado em Educação Física, correspondente ao ano de 2019, advindos do turno da manhã de uma Universidade particular localizada no bairro de Realengo, zona oeste do Rio de Janeiro, Brasil. Foram incluídos alunos, de ambos os sexos, com matrículas em vigência até o momento da coleta, condizentes com o perfil da amostra. Foram excluídos todos os alunos que estivessem enfermos e ou fazendo qualquer uso de medicamentos farmacológicos.

Este estudo foi realizado conforme as determinações do Conselho Nacional de Saúde [CNS] (Resolução n 466, 2012). Através da parceria Hospital Universitário Pedro Ernesto/Universidade do Estado do Rio de Janeiro, foi obtido o CAEE: 30464619.1.0000.5259 com data de aprovação final do CEP em 9 de abril de 2020 através da Plataforma 
Brasil. Todos os participantes assinaram o termo de consentimento livre e esclarecido (TCLE) antes de ingressar no estudo.

\section{Coleta de Dados}

A coleta de dados ocorreu em outubro de 2019 por intermédio de dois questionários autoadministrados e foi realizada por dois avaliadores nas salas de aula da instituição com os alunos que aceitaram participar voluntariamente da pesquisa e após o preenchimento do TCLE. Os questionários foram aplicados de forma transversal e respondidos individualmente pelos participantes do estudo.

\section{Escala de Humor}

Para mensurar o nível de humor dos participantes, foi aplicada a escala de humor de Brunel (Brunel Mood Scale - BRUMS), devidamente validada para uso no Brasil por Rohlfs et al. (2008) e que possui validação para aplicação em atletas e não atletas. A escala consiste em 24 perguntas fechadas a serem respondidas na escala ordinal, em que: 0 = nunca; 1 = um pouco. 2 = moderadamente; 3 = bastante e $4=$ extremamente.

\section{Escala de Percepção de Estresse - PSS14}

Na mensuração do nível de estresse, foi aplicada a escala de Luft et al. (2007), adequadamente validada parao Brasil. A escala, denominada Escala de Estresse Percebido (Perceived Stress Scale - PSS), busca mensurar o grau no qual os indivíduos percebem as situações como estressantes. Esta escala de percepção de estresse foi inicialmente apresentada com 14 itens (PSS 14). Cada item da escala possui opções de resposta que variam de zero a quatro ( 0 = nunca; 1 = quase nunca; 2 = às vezes; 3 = quase sempre; 4 = sempre). Os itens com conotação positiva $(4,5,6,7,9,10$ e 13) têm sua pontuação somada invertida, da seguinte maneira: $0=4 ; 1=3 ; 2$ $=2 ; 3=1$ e 4 = 0. Os demais itens possuem conotação negativa e devem ser somadas diretamente. $\bigcirc$ total da escala é a soma das pontuações destes 14 itens e os escores podem variar de zero a 56.

\section{Análise estatística}

Os dados foram tratados pelo programa IBM SPSS Statistics 20, versão 20 para Windows pela frequência das respostas, média e desvio padrão. A normalidade e a homogeneidade de variância dos dados da amostra foram verificadas pelos testes de Shapiro-Wilk. Foi calculado o coeficiente alfa de cronbach para determinar a confiabilidade das respostas. A associação entre as perguntas com as respostas foi analisada pelo teste do Qui-quadrado. As diferenças entre os agrupamentos das repostas foram analisadaspelo teste não paramétrico de Friedman. $O$ estudo adotou p<0,05 como nível de significância estatística.

\section{RESULTADOS}

A amostra foi composta por 38 estudantes universitários (idade: 25,51 \pm 4,24 anos). Os resultados do estudo são apresentados nas tabelas 1 e 2 com os dados descritivos das respostas aos questionários, e nos gráficos 1 e 2 pela associação entre as perguntas e respostas pelo teste do Qui-quadrado. 
Souza, F., Oliveira, J., Castro, J., Gama, D., \& Lima, V. (2022). Nível de estresse e humor em estudantes de Educação Física no último ano de curso. Revista Ciencias de la Actividad Física UCM, 23(1), enero-junio, 1-12. https://doi.org/10.29035/rcaf.23.1.2

Tabela 1

Resultados por perguntas do questionário de estresse (PSS 14).

\section{Nunca Quase nunca Às vezes Pouco freqüente Muito frequente Qui-quadrado}

\begin{tabular}{lll|llll} 
Q1 & 1 & 8 & 12 & 7 & 10 & 0,050 \\
\hline Q2 & 7 & 6 & 11 & 9 & 5 & 0,549 \\
\hline Q3 & 2 & 5 & 8 & 6 & 17 & 0,002 \\
\hline Q4 & 0 & 2 & 17 & 7 & 12 & 0,004 \\
\hline Q5 & 0 & 5 & 9 & 14 & 10 & 0,229 \\
\hline Q6 & 0 & 6 & 8 & 8 & 16 & 0,102 \\
\hline Q7 & 1 & 5 & 12 & 12 & 8 & 0,019 \\
\hline Q8 & 2 & 5 & 7 & 13 & 11 & 0,034 \\
\hline Q9 & 0 & 5 & 12 & 11 & 10 & 0,384 \\
\hline Q10 & 2 & 8 & 15 & 6 & 7 & 0,019 \\
\hline Q11 & 0 & 4 & 17 & 5 & 12 & 0,008 \\
\hline Q12 & 0 & 2 & 5 & 5 & 26 & 0,000 \\
\hline Q13 & 4 & 5 & 11 & 13 & 5 & 0,065 \\
\hline Q14 & 5 & 7 & 11 & 5 & 10 & 0,392 \\
\hline
\end{tabular}

Q: questão do questionário.

As repostas podem ser consideradas confiáveis, considerando o valor de 0,826 pelo cálculo de coeficiente alfa de Cronbach. O teste do Quiquadrado verificou associação entre as perguntas $e$ repostas Q1, Q3, Q4, Q7, Q10, Q11 e Q12.

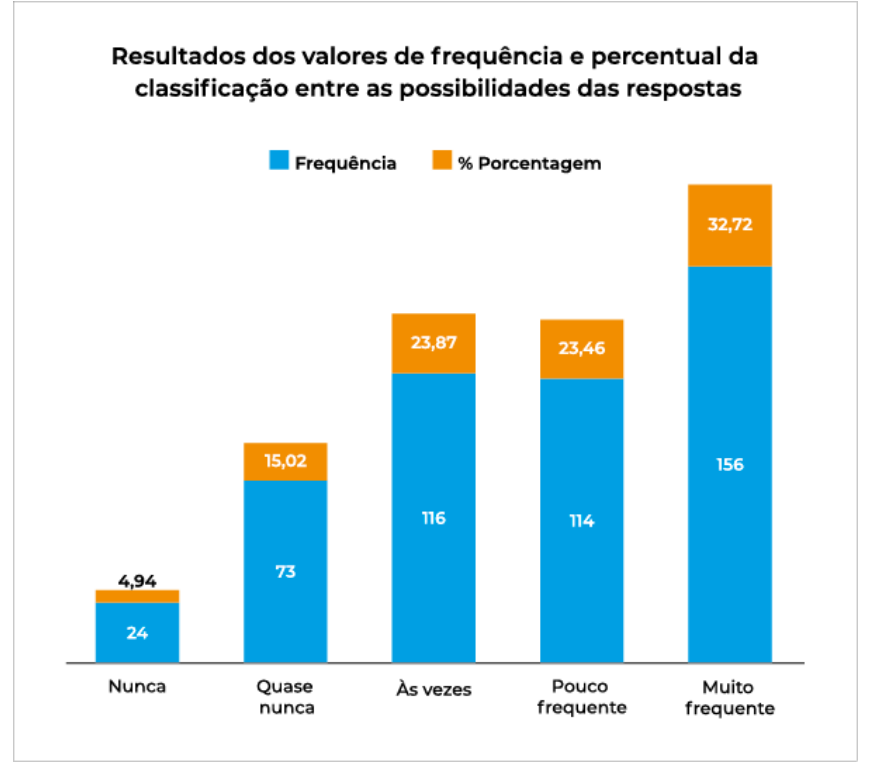

Gráfico 7. Resultados dos valores de frequência e percentual da classificação entre as possibilidades das respostas.

○ teste inferencial não paramétrico de Teste Friedman verificou que há diferença significativa entre as respostas $(p<0,001)$. Desta forma, a resposta "Muito Frequente", apresentou-se preponderante em relação às demais respostas. 
Souza, F., Oliveira, J., Castro, J., Gama, D., \& Lima, V. (2022). Nível de estresse e humor em estudantes de Educação Física no último ano de curso. Revista Ciencias de la Actividad Física UCM, 23(1), enero-junio, 1-12. https://doi.org/10.29035/rcaf.23.1.2

Tabela 2

Resultados das respostas da escala de humor.

\begin{tabular}{|c|c|c|c|c|c|c|}
\hline & Nada & Pouco & Moderadamente & Bastante & Extremamente & Qui-Quadrado \\
\hline Q1 & 27 & 2 & 4 & 2 & 3 & 0,00 \\
\hline Q2 & 3 & 9 & 11 & 11 & 4 & 0,10 \\
\hline Q3 & 12 & 13 & 4 & 5 & 4 & 0,03 \\
\hline Q4 & 7 & 4 & 5 & 12 & 10 & 0,20 \\
\hline Q5 & 28 & 1 & 3 & 4 & 2 & 0,00 \\
\hline Q6 & 16 & 5 & 5 & 7 & 5 & 0,02 \\
\hline Q7 & 14 & 9 & 8 & 3 & 4 & 0,04 \\
\hline Q8 & 7 & 7 & 6 & 10 & 8 & 0,88 \\
\hline Q9 & 12 & 15 & 6 & 2 & 3 & 0,00 \\
\hline Q10 & 11 & 9 & 8 & 5 & 5 & 0,47 \\
\hline Q11 & 25 & 6 & 2 & 2 & 3 & 0,00 \\
\hline Q12 & 26 & 4 & 5 & 0 & 3 & 0,00 \\
\hline Q13 & 9 & 8 & 3 & 8 & 10 & 0,43 \\
\hline Q14 & 6 & 8 & 5 & 9 & 10 & 0,69 \\
\hline Q15 & 6 & 5 & 11 & 10 & 6 & 0,43 \\
\hline Q16 & 24 & 8 & 4 & 2 & 0 & 0,00 \\
\hline Q17 & 23 & 5 & 4 & 3 & 3 & O,०० \\
\hline Q18 & 12 & 6 & 7 & 5 & 8 & 0,43 \\
\hline Q19 & 28 & 6 & 2 & 1 & 1 & O,०० \\
\hline Q20 & 1 & 10 & 12 & 9 & 6 & 0,05 \\
\hline Q21 & 5 & 6 & 6 & 10 & 11 & 0,43 \\
\hline Q22 & 22 & 4 & 8 & 2 & 2 & O,०০ \\
\hline Q23 & 7 & 6 & 10 & 6 & 9 & 0,78 \\
\hline Q24 & 18 & 5 & 9 & 3 & 3 & 0,00 \\
\hline
\end{tabular}

Q: questão do questionário.

O teste do Qui-quadrado para os resultados das respostas da escala de humor verificou associação entre as perguntas e repostas: Q1, Q3, Q5, Q6, Q7, Q9, Q11, Q12, Q16, Q17, Q19, Q20, Q22 e Q24. 
Resultados dos valores de frequência e percentual da classificação entre as possibilidades das respostas

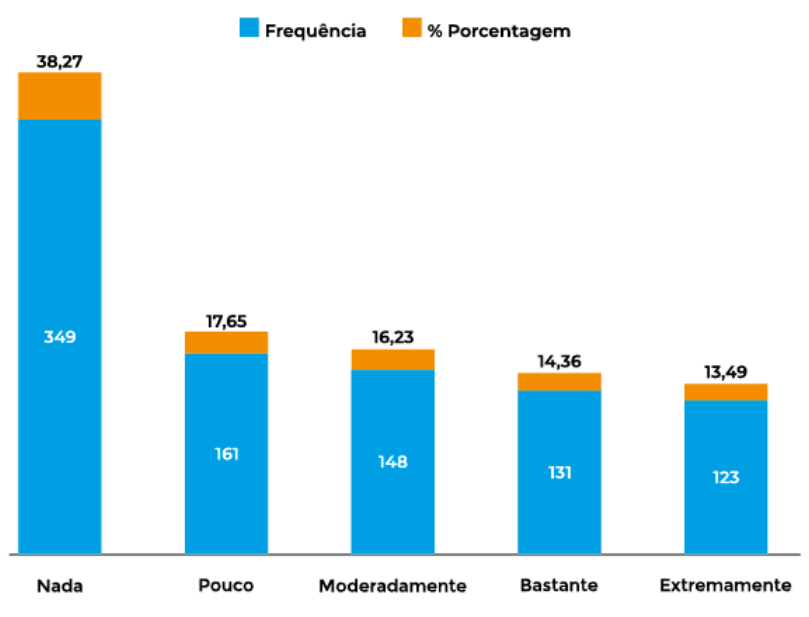

Gráfico 2. Resultados dos valores de frequência e percentual da classificação entre as possibilidades das respostas.

○ teste inferencial não paramétrico de Friedman verificou que há diferença significativa entre as respostas $(p=0,026)$. Desta forma, a resposta "Nada" foi preponderante em relação às demais respostas.

\section{DISCUSSÃO}

O universo acadêmico parece ser um ambiente que pode promover problemas à saúde mental dos alunos (Graner \& Cerqueira, 2019). O último ano da graduação é uma etapa decisiva para o futuro dos estudantes. Essa etapa de conclusão traz consigo algumas tarefas, que demandam dedicação às obrigações acadêmicas. Nessa fase, o estudante precisa cumprir as horas de estágio supervisionado obrigatório, realizar as últimas disciplinas da grade curricular e produzir o trabalho de conclusão de curso (TCC), que geralmente representa um trabalho de grande dificuldade para a maioria dos estudantes.

Os dados obtidos no presente estudo corroboram com a hipótese de que estudantes de graduação, em especial no ultimo ano de graduação, estão expostos a agentes estressores que podem ser advindos do ambiente acadêmico. Os alunos não apresentaram alterações negativas relacionadas ao humor, já que foi identificada uma diferença significativa entre as respostas relatadas na escala de Brunel. A resposta "Nada" apareceu com maior frequência (38,27\%), o que pode ser sustentado pela constante variação do humor e pelas diferentes representações de um momento que podem refletir na autopercepção do humor. Esses resultados convergem com os achados de Castro et al. (2017), que verificaram que os universitários dos períodos mais avançados podem apresentar dificuldade em conciliar estágios, disciplinas acadêmicas e a escrita do TCC.

O estresse em estudantes de Educação Física também foi evidenciado por Kumar \& Bhukar (2013), que procuraram mensurar o nível de estresse, bem como estratégias de enfrentamento dos estudantes de Educação Física e Engenharia pertencentes a duas universidades na Índia. Foram selecionados 60 estudantes, dos quais 30 correspondiam ao curso de Educação Física e 30 ao curso de Engenharia. O questionário Compulsive, time-urgentand aggressive behavior (Girdano \& Everly, 1979) foi utilizado para analisar o nível de estresse eas estratégias de enfrentamento foram avaliadas através do questionário Coping strategy (Heyward, 1991). O estudo verificou que o nível de estresse em mulheres foi maior do que nos homens independentemente do curso e as estratégias de enfrentamento dos homens foram significativamente maiorquando comparadas as das mulheres. No curso de Educação Física, 80\% dos homens e 68\% das mulheres apresentaram uma estratégia de enfrentamento considerada adequada ou boa, enquanto no curso de Engenharia, 62\% dos homens e 50\% das mulheres apresentaram uma estratégia de enfrentamento considerada adequada ou boa. Ademais, em ambos os cursos, nenhum dos alunos obteve uma estratégia de enfrentamento considerada excelente.

Mussi et al. (2019) realizaram um estudo transversal, comparando níveis de estresse de 150 
estudantes de Enfermagem do primeiro e do último ano. Os resultados mostram que os níveis de estresse dos alunos do último ano foram maiores em comparação aos do primeiro ano. Abu-Ghazaleh et al. (2016) encontraram resultados similares ao realizarem um estudo longitudinal com 135 alunos de odontologia do primeiro ano de curso e 67 estudantes do quinto ano do curso, comparando os níveis de estresse dos mesmos. De acordo com os resultados, os níveis de estresse aumentaram ao longo do curso, havendo uma diferença significante entre os resultados. Alunos no quinto ano de curso demonstraram níveis de estresse significativamente mais altos do que no primeiro ano.

Enns et al. (2018) realizaram um estudo transversal com 203 estudantes universitários de psicologia, enfermagem e serviço social. Os resultados sugerem que quanto mais inteligência emocional, menor o nível de estresse percebido. Essa associação foi mediada por respostas de enfrentamento adaptativas e desadaptativas. Já Alyami et al. (2017), em um estudo transversal feito com 214 estudantes de psicologia, relataram estresse nos estudantes, cuja prevalência de estresse foi de 71\%. Entretanto, o estresse não se mostrou relacionado ao nível de desempenho acadêmico.

A demanda de evidências do estresse em estudantes acadêmicos provoca estudos com possiveis intervenções sobre a problemática. Shearer et al. (2016) realizaram uma intervenção com 74 estudantes universitários para verificar os efeitos da meditação mindfulness nos níveis de estresse. Os participantes foram divididos em grupo experimental, grupo controle ativo (em que os participantes tinham contato com cães entre as pausas do estudo) e grupo controle sem tratamento. Os resultados sugerem que, enquanto os participantes do grupo controle ativo não se diferenciaram do grupo controle sem tratamento, os alunos que participaram da meditação mindfulness mostraram respostas mais adaptativas ao estresse. $O$ estudo sugere que o treinamento de meditação mindfulness pode auxiliar os alunos a gerenciarem melhor o estresse. Os achados de Shearer et al. (2016) corroboram com a pesquisa de Regehr et al. (2013), uma metanálise com 24 estudos, envolvendo 1431 estudantes, onde foram investigadas intervenções para reduzir estresse em estudantes universitários. Os resultados sugerem que intervenções cognitivas baseadas em comportamento e mindfulness focadas na redução do estresse reduzem significativamente os sintomas de ansiedade. Os desfechos secundários incluíram níveis mais baixos de depressão e cortisol.

Apesar do nível de estresse elevado, no presente estudo, os participantes não apresentaram perda de humor, o que foi averiguado pela BRUMS. Ogurlu (2015) buscou verificar a relação humor x aprendizado e possíveis intervenções para melhorar o humor. $\bigcirc$ autor utilizou uma amostra composta por 319 estudantes de graduação com o objetivode examinar as relações entre inteligência cognitiva, inteligência emocional e estilos de humor. 0 questionário sobre estilos de humor The Schutte Revised, Inteligência Emocional e Raven Standard Progressive Matrices Test Plus foram utilizados para obter os dados. Os resultados indicaram que a inteligência emocional está positivamente correlacionada com 0 humor. A inteligência cognitiva não teve relação estatisticamente significativa com os estilos de humor, exceto o estilo de humor afiliativo em que o humor é utilizado para promover relacionamentos com os outros.

Annesi et al. (2017) analisaram estudantes universitários que apresentaram nível de atividade física abaixo do recomendado, em que a amostra consistiu de 52 estudantes, sendo 69\% do sexo feminino, matriculados em uma matéria eletiva de atividade física e 32 estudantes, sendo 69\% do sexo feminino que não estavam matriculados nessa matéria. $\bigcirc$ estudo buscou avaliar a associação entre estudantes universitários e a prática de atividade física, em que cursos com 4 ou mais anos de duração oferecem cursos eletivos em condicionamento físico (por exemplo: treinamento com pesos) ou esportes (por exemplo: tênis), correlacionando com possíveis alterações no humor. Os estados de humor foram 
mensurados por uma Escala de Distúrbios do Perfil dos Estados de Humor através de um formulário. Os achados indicam que os alunos matriculados obtiveram melhora no humor e indicam uma possível contribuição para melhoria da saúde mental e sucesso acadêmico.

Durante a execução da pesquisa, algumas limitações foram identificadas, como a falta de dados com estudantes dos primeiros períodos, a fim de comparar os níveis de estresse e verificar se, de fato, esses níveis são maiores no último período; a aplicação da escala de humor uma única vez, não levando em conta a não rigidez do estado de humor, visto que o humor pode oscilar de acordo com os acontecimentos do dia a dia, podendo sofrer influência de fatores, como contexto familiar, condições financeiras e rede de amizades. Desta forma, sugerimos novas pesquisas com estudos longitudinais, maior número de participantes, a aplicação das escalas com alunos de outros períodos a fim de comparar os níveis de estresse, aplicar a escala de humor ao longo do período estudado e inclusão de marcadores bioquímicos para avaliar os níveis de estresse.

\section{CONCLUSÃO}

Conclui-se que os estudantes no último ano do curso de bacharelado em Educação Física apresentam nivel elevado de estresse, sem resultado para humor negativo. As descobertas são importantes para o embasamento de possíveis intervenções futuras que visem reduzir o estresse e o humor negativo, melhorando a qualidade de vida de estudantes e futuros profissionais de Educação Física, gerando um ambiente mais saudável, que permita o amplo desenvolvimento profissional, diminuindo os riscos de potenciais fontes de adoecimento. Uma estratégia efetiva para minimizar os efeitos do estresse sobre os estudantes em fase final de curso é o oferecimento de serviços permanentes de terapia psíquica pelas administrações universitárias.

\section{REFERÊNCIAS}

Abu-Ghazaleh, S.B., Sonbol, H.N., \& Rajab, L.D. (2016). A longitudinal study of psychological stress among undergraduate dental students at the University of Jordan. BMC Medical Education, $\quad 90$. https://doi.org/10.1186/s12909-016-0612-6

Alyami, M., Melyani, Z., Johani, A. A., Ullah, E., Alyami, H., Sundram, F., Hill, A., \& Henning, M. (2017). The impact of self-esteem, academic selfefficacy and perceived stress on academic performance: A cross-sectional study of Saudi psychology students. European Journal of Educational Sciences, 4(3), 51-63. http://dx.doi.org/10.19044/ejes.v4no3a5

Annesi, J. J., Porter, K. J., Hill, G. M., \& Goldfine, B. D. (2017). Effects of instructional physical activity courses on overall physical activity and mood in university students. Research Quarterly for Exercise and Sport, 88(3), 358364. https://doi.org/10.1080/02701367.2017.13362 80

Asayesh, H., Sharififard, F., Mosavi, M., Kharameh, Z. T., Arani, Z. A., \& Bidgoli, A. S. (2016). Correlation among academic stress, academic burnout, and academic performance in nursing and paramedic students of Qom University of Medical Sciences, Iran. Qom University of Medical Sciences, 10(7), 74-83. http://journal.muq.ac.ir/article-7-491-fa.html

Castro, J. B. P., Vale, R. G. S., Aguiar, R. S., \& Mattos, R. S. (2017). Perfil do estilo de vida de universitários de Educação Física da cidade do Rio de Janeiro. Revista Brasileira de Ciência e Movimento, 25(2), 73-83. https://pesquisa.bvsalud.org/portal/resourc e/fr/biblio-882131

Eldar, E., Rutledge, R. B., Dolan, R. J., \& Niv, Y. (2016). Mood as representation of momentum. Trends in Cognitive Sciences, 20(1), 15-24. https://doi.org/10.1016/j.tics.2015.07.010 
Enns, A., Eldridge, G. D., Montgomery, C., \& Gonzalez, V. M. (2018). Perceived stress, coping strategies, and emotional intelligence: A cross-sectional study of university students in helping disciplines. Nurse Education Today, 68 226-231. https://doi.org/10.1016/j.nedt.2018.06.012

Frazier, P., Gabriel, A., Merians, A., \& Lust, K. (2019). Understanding stress as an impediment to academic performance. Journal of American College Health, 67(6), 562-570. https://doi.org/10.1080/07448481.2018.14996 49

Girdano, D. A., \& Everly, G. S. (1979). Controlling stress and tension: A Holistic Approach. Prentice Hall.

Graner, K. M., \& Cerqueira, A. T. R. (2019). Integrative review: psychological distress among university students and correlated factors. Ciência \& Saúde Coletiva, 24(4), 1327-1346. https://doi.org/10.1590/1413-

81232018244.09692017

Greeley, B., \& Seidler, R. D. (2017). Mood induction effects on motor sequence learning and stop signal reaction time. Experimental Brain Research, 235, 47-56 https://doi.org/10.1007/s00221-016-4764-8

Gustems-Carnicer, J., Calderón, C., \& CalderónGarrido, D. (2019). Stress, coping strategies and academic achievement in teacher education students. European Journal of Teacher Education, 42(3), 375-390. https://doi.org/10.1080/02619768.2019.15766 29

Heyward, V. H. (1991). Advanced fitness Assessment and exercise Prescription. Human Kinetics Books.

Huang, N. T., \& Lee, H. L. (2019). Ability emotional intelligence and life satisfaction: Humor style as a mediator. Social Behavior and Personality: An International Journal, 47(5), 1-13. https://doi.org/10.2224/sbp.7805
Kluen, L. M., Nixon, P., Agorastos, A., Wiedemann, K., \& Schwabe, L. (2017). Impact of stress and glucocorticoids on schema-based learning. Neuropsychopharmacology, 42, 1254-1261. https://doi.org/10.1038/npp.2016.256

Kumar, S., \& Bhukar, J. P. (2013). Stress level and coping strategies of college students. Journal of Physical Education and Sport Management, $\quad 4(1), \quad 5-11$. https://academicjournals.org/article/article1 379492114_Kumar\%20and\%20Bhukar.pdf

Lee, W. W. S. (2017). Relationships among grit, academic performance, perceived academic failure, and stress in associate degree students. Journal of Adolescence, 60 , 148-152. https://doi.org/10.1016/j.adolescence.2017.08 .006

Lindau, M., Almkvist, O., \& Mohammed, A. H. (2016). Effects of stress on learning and memory. En G. Fink (Ed.), Stress: Concepts, cognition, emotion, and behavior (1ª Ed., Vol. 1, pp. 153160). Elsevier Academic Press. https://doi.org/10.1016/C2013-0-12842-5

Luft, C. D. B., Sanches, S. O., Mazo, G. Z., \& Andrade, A. (2007). Versão brasileira da Escala de Estresse Percebido: tradução e validação para idosos. Revista de Saúde Pública, 47(4), 606-615. http://dx.doi.org/10.1590/5003489102007000400015

Lujan, H. L., \& DiCarlo, S. E. (2016). Humor promotes learning!. Advances in physiology education, 40(4), 433-434. https://doi.org/10.1152/advan.00123.2016

Moutinho, I. L. D., Maddalena, N. C. P., Roland, R. K., Lucchetti, A. L. G., Tibiriçá, S. H. C., Ezequiel, O. D. S., \& Lucchetti, G. (2017). Depression, stress and anxiety in medical students: A cross-sectional comparison between students from different semesters. Revista da Associação Médica Brasileira, 63(1), 2128 http://dx.doi.org/10.1590/18069282.63.01.21 
Mussi, F. C., Pires, C. G. S., Carneiro, L. S., Costa, A. L. S., Ribeiro, F. M. S. S., \& Santos, A. F. (2019). Comparison of stress in freshman and senior nursing students. Revista da Escola de Enfermagem da USP, 53, e03431. https://doi.org/10.1590/s1980$220 \times 2017023503431$

Ng, K. C., Chiu, W. K., \& Fong, B. Y. F. (2016). A review of academic stress among Hong Kong undergraduate students. Journal of Modern Education Review, 6(8), 531-540. https://doi.org/10.15341/jmer(21557993)/08.06.2016/003

Ogurlu, Ü. (2015). Relationship between cognitive intelligence, emotional intelligence and humor styles. International Online Journal of Educational Sciences, 7(2), 15-25. https://iojes.net/?mod=tammetin\&makalea di=\&makaleur|=|OJES_1651.pdf\&key=40954

Regehr, C., Glancy, D., \& Pitts, A. (2013). Interventions to reduce stress in university students: A review and meta-analysis. Journal of Affective Disorders, 148(1), 1-11. https://doi.org/10.1016/j.jad.2012.11.026

Resolução n 466 de 2012 [Conselho Nacional de Saúde]. Conselho Nacional de Saúde aprovar as seguintes diretrizes e normas regulamentadoras de pesquisas envolvendo seres humanos. 13 de junio de 2013.

https://conselho.saude.gov.br/resolucoes/2 012/Reso466.pdf

Rohlfs, I. C. P. M., Rotta, T. M., Luft, C. D. B., Andrade, A., Krebs, R. J., \& Carvalho, T. (2008). A Escala de Humor de Brunel (BRUMS): instrumento para detecção precoce da síndrome do excesso de treinamento. Revista Brasileira de Medicina do Esporte, 14(3), 176-181. https://doi.org/10.1590/S151786922008000300003

Saleh, D., Camart, N., \& Romo, L. (2017). Predictors of Stress in College Students. Frontiers in psychology, 8 , 19. https://doi.org/10.3389/fpsyg.2017.00019
Savage, B. M., Lujan, H. L., Thipparthi, R. R., \& DiCarlo, S. E. (2017). Humor, laughter, learning, and health! A brief review. Advances in Physiology Education, 47(3), 347-347. https://doi.org/10.1152/advan.00030.2017

Shankar, N. L., \& Park, C. L. (2016). Effects of stress on students' physical and mental health and academic success. International Journal of School \& Educational Psychology, 4(1), 5-9. https://doi.org/10.1080/21683603.2016.113053 2

Shearer, A., Hunt, M., Chowdhury, M., \& Nicol, L. (2016). Effects of a brief mindfulness meditation intervention on student stress and heart rate variability. International Journal of Stress Management, 23(2), 232-254. https://doi.org/10.1037/a0039814

Smith, S. M., \& Vale, W. W. (2006). The role of the hypothalamic-pituitary-adrenal axis in neuroendocrine responses to stress. Dialogues in Clinical Neuroscience, 8(4), 383-395 https://doi.org/10.31887/DCNS.2006.8.4/ssm ith

Thomas, J. R., Nelson, J. K., \& Silverman, S. J. (2012). Métodos de pesquisa em atividade física (6a Ed.). Artmed.

Tsigos, C., \& Chrousos, G. P. (2002). Hypothalamicpituitary-adrenal axis, neuroendocrine factors and stress. Journal of Psychosomatic Research, 53(4), 865-871. https://doi.org/10.1016/500223999(02)00429-4

Tsukawaki, R., \& Imura, T. (2020). Preliminary Verification of Instructional Humor Processing Theory: Mediators Between Instructor Humor and Student Learning. Psychological reports, 123(6), 2538-2550. https://doi.org/10.1177/0033294119868799

Wingenfeld, K., \& Wolf, O. T. (2014). Stress, memory, and the hippocampus. Frontiers of Neurology and Neuroscience, 34, 109-120. https://doi.org/10.1159/000356423 
Yaribeygi, H., Panahi, Y., Sahraei, H., Johnston, T. P., \& Sahebkar, A. (2017). The impact of stress on body function: A review. EXCLI Journal, 76, 1057-1072. https://doi.org/10.17179/excli2017480

\section{Dirección para correspondencia}

Fernanda Alves de Souza

Grupo de Pesquisa em Biodinâmica do

Desempenho, Exercício e Saúde (BIODESA)

Universidade Castelo Branco.

Rio de Janeiro, Brasil.

Dirección postal: Avenida Santa Cruz, 1631, Realengo,

Rio de Janeiro - RJ, Brasil.

CEP: $21710-255$

ORCID: https://orcid.org/0000-0002-2424-6860

Contacto: nanda10rj@hotmail.com

Recibido: 03-08-2021

Aceptado: 29-10-2021 